\title{
Anti-inflammatory T-cell shift in neuropathic pain
}

\author{
Benjamin Luchting*, Banafscheh Rachinger-Adam, Jens Heyn, Ludwig Christian Hinske, Simone Kreth \\ and Shahnaz Christina Azad
}

\begin{abstract}
Background: The classification of pain into nociceptive and neuropathic pain is based on characteristic symptoms and different pathophysiological mechanisms. In a recent investigation, we found a disrupted TH17/Treg balance in patients suffering from chronic unspecific low back pain (CLBP). These patients did not show any signs of neuropathy. There is evidence for a considerable impact of the immune system also in neuropathic pain. However, the role of the adaptive immune system is still unclear. In the present study, we investigated systemic T-cell subset responses and T-cell related cytokine profiles in patients with chronic neuropathic pain.

Methods: We analyzed T-cell subsets, mRNA expression and T-cell-related cytokine profiles in 26 patients suffering from neuropathic pain in comparison to 26 healthy controls. Using multicolor flow cytometry (FACS), we quantified the number of Thelper cells 1 (TH1), TH2, TH17 and regulatory T-cells (Tregs). Forkhead-Box-Protein 3 (FoxP3), Transforming growth factor- $\beta$ (TGF- $\beta$ ) and RAR-related orphan receptor- $\gamma T$ (ROR- $\gamma$ T) mRNA expression was determined by quantitative real-time PCR ( $(\mathrm{PCR}$ ) and levels of pain-related cytokines were measured by Human Cytokine Multiplex Immunoassay (Macrophage inflammatory protein-1a (MIP-1a), Tumor necrosis factor-a (TNF-a), Interferon- $\gamma($ IFN- $\gamma)$, Interleukin (IL) -4, IL-6, IL-10, IL-17, and IL-23).

Results: We found a TH17/Treg imbalance with significantly increased anti-inflammatory Tregs and decreased pro-inflammatory TH17 cells in patients with neuropathic pain as compared to healthy controls. These results were confirmed on mRNA level: Treg-related FoxP3 and TGF- $\beta$ mRNA expression was elevated, whereas expression of TH17-related RORYT was reduced. Cytokine analyses revealed only marginal changes.
\end{abstract}

Conclusions: Our investigation revealed a clear shift of T-cell subsets towards anti-inflammation in patients with neuropathic pain. Interestingly, this is quite similar to our previous findings in CLBP patients, but even more pronounced. Therefore, it remains to be elucidated in future investigations whether the immune changes represent an underlying pathophysiological mechanism or an epiphenomenon induced by ongoing pain and stress.

German Clinical Trial Register (DRKS): Trial registration number: DRKS00005954

Keywords: Neuropathic pain, T-cells, TH17, Treg, Cytokines, Neuroinflammation

\section{Background}

Neuropathic pain represents a major problem in clinical practice. In contrast to nociceptive pain, which is caused by damage or potential damage to tissue, neuropathic pain occurs due to a lesion or disease of the peripheral or central nervous system. It is characterized by burning and lancinating pain sensations and further somatosensory disturbances like hypo- and hypersensitivity. Very often, neuropathic pain is more difficult to treat and more refractory to common analgesics, including non-steroidal

\footnotetext{
* Correspondence: benjamin.luchting@med.uni-muenchen.de Department of Anesthesiology and Pain Medicine, Ludwig-Maximilians University Munich, Marchioninistr. 15, 81377 Munich, Germany
}

anti-inflammatory drugs and opioids, than nociceptive pain $[1,2]$. Despite extensive research, the underlying pathophysiological mechanisms of neuropathic pain are still not fully understood. In recent years, increasing evidence indicates a pivotal role of the immune system in neuropathic pain $[3,4]$. The majority of previously published data link pain syndromes with higher levels of pro-inflammatory cytokines. Due to these findings, attempts were made in numerous studies to reduce neuropathic pain by blocking pro-inflammatory or enhancing anti-inflammatory immune cells and cytokines [3]. For example, in animal models of neuropathy, tumor necrosis factor- $\alpha$ (TNF- $\alpha$ ), Interleukin (IL) -6, IL-17 and 
Makrophage inflammatory protein1- $\alpha$ (MIP1- $\alpha$ ) blockers reduced pain hypersensitivity [5-9]. Comparable results were obtained by increasing the anti-inflammatory cytokines IL-4, IL-10 or Transforming growth factor- $\beta$ (TGF- $\beta$ ) [10-12]. Nevertheless, despite those promising experimental findings, there are no pharmacological agents available for the specific immunological therapy of neuropathic pain until now.

Cytokines and neutrophils are important during the early stages of acute pain, whereas T-lymphocytes seem to play a central role in chronic neuropathic pain [13]. Regarding T-cells as key players of the adaptive immune system, a TH1/TH2 imbalance has already been shown in patients with complex regional pain syndrome (CRPS) and chronic pelvic pain $[14,15]$. In recent years, TH1/ $\mathrm{TH} 2$ dichotomy has been extended by the identification of two other $\mathrm{CD}_{4}^{+} \mathrm{T}$-cell lineages: TH17 and regulatory T-cells (Tregs) [16]. TH17 cells appear to be the key effector T-cells in a variety of human autoimmune diseases and Tregs play a vital role in controlling adaptive immune responses. In neuropathy, TH17 has been linked to increased pain sensitivity and destructive effects promoting persistent pain [16], while Tregs were found to be mainly involved in the endogenous recovery [17]. Recently published data showed an increased proportion of Tregs in patients with postherpetic neuralgia [18]. Assuming a beneficial role for an anti-inflammatory T-cell shift, a phase one trial was carried out with an anti-CD28 antibody, preferentially expanding $\mathrm{TH} 2$ cells and Tregs in human volunteers. Despite promising results in several animal models, the clinical trial had to be cancelled because of severe side effects [19]. In patients with neuropathic pain, the role of $\mathrm{T}$-cell subsets has not yet been investigated.

In a recent study, we found that patients suffering from nociceptive, non-specific chronic low back pain (CLBP), without any signs of neuropathic pain components, display a clear disruption of the TH17/Treg balance as compared to healthy volunteers [20]. Since clinical and pathophysiological mechanisms differ considerably between nociceptive and neuropathic pain, we aimed to detail changes of pro- and anti-inflammatory $\mathrm{T}$-cell subsets and the respective relative mRNA expression, as well as pain-related cytokine levels in patients with chronic neuropathic pain in comparison to pain free controls. While the cytokine measurement did not reveal any relevant results, we found a distinct anti-inflammatory shift of the T-cell subsets and their respective mRNA expression.

\section{Methods}

\section{Ethics statement}

The study followed the principles of the Declaration of Helsinki and was approved by the Ethics Committee of the Ludwig Maximilians University Munich (Ethical approval number: 331-10). This study was registered on German Clinical Trial Register (Trial registration: DRKS00005954).

\section{Subjects}

Patient recruitment of our prospective study was estimated to last for two years. All patients presented to our Department of Anesthesiology and Pain Medicine, LudwigMaximilians University Munich with neuropathic pain for at least six months were assessed for fulfillment of the inclusion criteria and asked for their consent to participate in the study. In addition, healthy pain-free volunteers without any signs or history of pain were asked for their participation. Neuropathic pain was diagnosed according to its international definition: 'pain caused by a lesion or disease of the somatosensory nervous system' [2], by pain history, physical examination and the PainDETECT questionnaire.

This questionnaire consists of several items related to neuropathic symptoms (burning sensations, tingling or prickling sensations, shooting or lancinating, hyperalgesia, dysesthesia, allodynia or paresthesia) with excellent sensitivity (85\%) and specificity (80\%) [21]. Additionally, quantitative sensory testing (QST) was performed in all patients according to the protocol of the German Research Group on neuropathic pain [22]. Patients with mixed pain (nociceptive and neuropathic components) like complex regional pain syndrome (CRPS) and low back pain with radiculopathy were excluded. Further exclusion criteria were autoimmune, chronic systemic, inflammatory, neoplastic or psychiatric diseases, as well as drug abuse and pregnancy. Patients taking any current medication with opioids, non-opioids or co-analgesics were excluded. None of the patients had been treated with corticosteroids or had received immunomodulatory agents currently or in the past. Any signs of acute inflammatory disease were disclosed by laboratory examination, including plasma concentration of $\mathrm{C}$-reactive protein (CRP), total and differential leucocyte count, as well as measurement of the body temperature. Patients rated their recalled average pain intensity using an 11-point numerical rating scale (NRS): 0 meaning 'no pain' and 10 meaning 'worst pain imaginable'.

Self-perceived stress was evaluated using the German version of the Questionnaire for Actual Demands (KAB: Kurzfragebogen zur aktuellen Beanspruchung) in patients and healthy volunteers. The KAB was designed to repeatedly quantify an individual's acute or chronic stress. It is highly sensitive to short-term or situational changes during a stressful experience. The rating is based on a six-point scale ranging from one to six based on normalized adjectives. Higher KAB values indicate increased perceived levels of stress [23]. 


\section{Cytokine assessment}

Samples of peripheral blood from all patients and healthy controls were collected between 9:00 and 9:30 am, centrifuged at $2000 \times \mathrm{g} / 10 \mathrm{~min}$ and stored in polypropylene aliquot tubes at $-80^{\circ} \mathrm{C}$. Samples were then assessed for levels of T-cell-related cytokines using a human cytokine multiplex immunoassay (Myriad Rules-Based Medicine Inc., Austin, Texas, United States). The multiplex microbead assay is based on Luminex technology and measures proteins in a similar manner to standard sandwich ELISA, with comparable sensitivity and range. Regarding the detection limits, the lower limit of quantitation (LLOQ) for the cytokines were: MiP1- $\alpha$ : $42.0 \mathrm{pg} / \mathrm{ml}$, TNF- $\alpha: 23.0 \mathrm{pg} / \mathrm{ml}$, IFN- $\gamma: 1.5 \mathrm{pg} / \mathrm{ml}$, IL-4: $29.0 \mathrm{pg} / \mathrm{ml}$, IL-6: 11.0 pg/ml, IL-10: $6.9 \mathrm{pg} / \mathrm{ml}$, IL-17: $4.0 \mathrm{pg} / \mathrm{ml}$, and IL-23: $0.59 \mathrm{pg} / \mathrm{ml}$. The LLOQ is the lowest concentration of an analyte in a sample that can be reliably detected and at which the total error meets the laboratory's requirements for accuracy [24].

\section{Flow cytometric staining and analysis}

Peripheral blood mononuclear cells (PBMCs) were separated by density gradient preparation over Ficoll-Uropoline (Sigma Aldrich, Taufkirchen, Germany) of all heparinized venous blood samples. Then, PBMCs were cryopreserved in Roswell Park Memorial Institute medium (RPMI) freezing media (Sigma Aldrich, Taufkirchen, Germany), containing 10\% Fetal calf serum (FCS), (Sigma Aldrich, Taufkirchen, Germany) and 10\% Dimethyl sulfoxide (DMSO), (Sigma Aldrich, Taufkirchen, Germany) [25], and stored at $-30^{\circ} \mathrm{C}$ for 24 hours, and then at $-196^{\circ} \mathrm{C}$ until measurement. After storage, samples were thawed rapidly and washed twice to eliminate DMSO. For TH1, TH2 and TH17 analysis, cells were stimulated for five hours with cell stimulation cocktail, including protein transport inhibitors Phorbol 12-myristate 13-acetate (PMA), ionomycin, Brefeldin A and monensin (eBioscience, San Diego, California, United States), according to the manufacturer's protocol. Subsequently, cells were extracellularly stained with anti-human CD4 antibody and consecutively fixed and permeabilized (Fix-Perm-Solutions A and B, Life Technologies, Darmstadt, Germany) for intracellular staining with anti-human Interferon- $\gamma$, Interleukin (IL) -4 and IL-17 antibody (Biolegend, San Diego, California, United States). T-cell distribution was measured by fluorescent-activated cell sorting (FACS) analysis with the Attune Acoustic Focusing Cytometer (Life Technologies, Carlsbad, United States), and exemplary pictures of the gating strategy for TH17 cells are displayed in Figure 1 and Additional files 1 and 2. Tregs were identified and quantified after surface staining of PBMCs with monoclonal antibodies (mAbs) specific for anti-human CD4, CD25 and CD127 and intracellular staining with an anti-human FoxP3 antibody (Biolegend, San Diego, California, United States). The frequencies of $\mathrm{CD} 4^{+} \mathrm{CD} 25^{\text {high }} \mathrm{T}$-cells and
$\mathrm{CD} 4{ }^{+} \mathrm{CD} 25^{\text {high }} \mathrm{CD} 127^{\text {low }} \mathrm{FoxP}^{+} \mathrm{T}$-cells were expressed as percentage of total $\mathrm{CD} 4^{+} \mathrm{T}$-cells by sequential gating on lymphocytes. Exemplary pictures of the gating strategy for Tregs are displayed in Figure 2 and Additional files 1 and 2. Isotype controls (Biolegend, San Diego, California, United States) were given for compensation and confirmation of antibody specificity.

\section{Quantitative real-time PCR (qPCR)}

$\mathrm{CD} 4^{+}$cells were isolated from $\mathrm{PBMCs}$ by magnetic separation with Whole Blood CD4 MicroBeads (MACS Miltenyi Biotec, Auburn, California, United States) according to the manufacturer's recommendations. Subsequently, total RNA was isolated using the mirVana miRNA Isolation Kit followed by a DNase-digest with Turbo DNA-free Kit (Ambion, Darmstadt, Germany). Quantity and purity of the isolated RNA were measured using a NanoDrop ND-1000 spectrophotometer (Peqlab, Erlangen, Germany). After amplification of total RNA using TargetAmp 1-Round aRNA Amplification Kit (Epicentre Biotechnologies, Madison, Wisconsin, United States) and purification using RNeasy Mini Kit (Qiagen, Hilden, Germany), cDNA synthesis was performed with SuperScript III First Strand Synthesis System (Invitrogen, Darmstadt, Germany) and random hexamers (Qiagen, Hilden, Germany). Quantitative RT-PCR was performed in duplicates with the LightCycler 480 instrument (Roche Diagnostics, Mannheim, Germany) using LightCycler 480 Probes Master and RealTime ready single assays (Roche Diagnostics, Mannheim, Germany) and UniversalProbeLibrary (UPL) probes. The RealTime ready single assays contain target-specific primers and a UPL-LNA probe (Roche Diagnostics, Mannheim, Germany). Primer sequences and qPCR characteristics are given in Table 1. The cycling conditions comprised an initial denaturation phase at $95^{\circ} \mathrm{C}$ for 10 minutes, followed by 45 cycles at $95^{\circ} \mathrm{C}$ for 10 seconds, $60^{\circ} \mathrm{C}$ for 30 seconds and $72^{\circ} \mathrm{C}$ for one second. Relative mRNA expression of FoxP3, TGF- $\beta$ and ROR $\gamma$ T was calculated by Relative Quantification Software (Roche Diagnostics, Mannheim, Germany) using an efficiency-corrected algorithm with standard curves and reference gene normalization against the reference genes succinate dehydrogenase complex subunit A (SDHA) and TATA box binding protein (TBP) as described in Ledderose et al. [26].

\section{Statistical analyses}

Statistical analyses were performed using SigmaStat 12.0 (Systat Software, Chicago, United States). Every statistical analysis was started with testing for normal distribution using the Shapiro-Wilk test. Testing for differences between groups was accomplished by the $T$-test for all data with normal distribution and the nonparametric Mann-Whitney rank sum test for all data without normal distribution. Family-wise error rate was controlled at a 

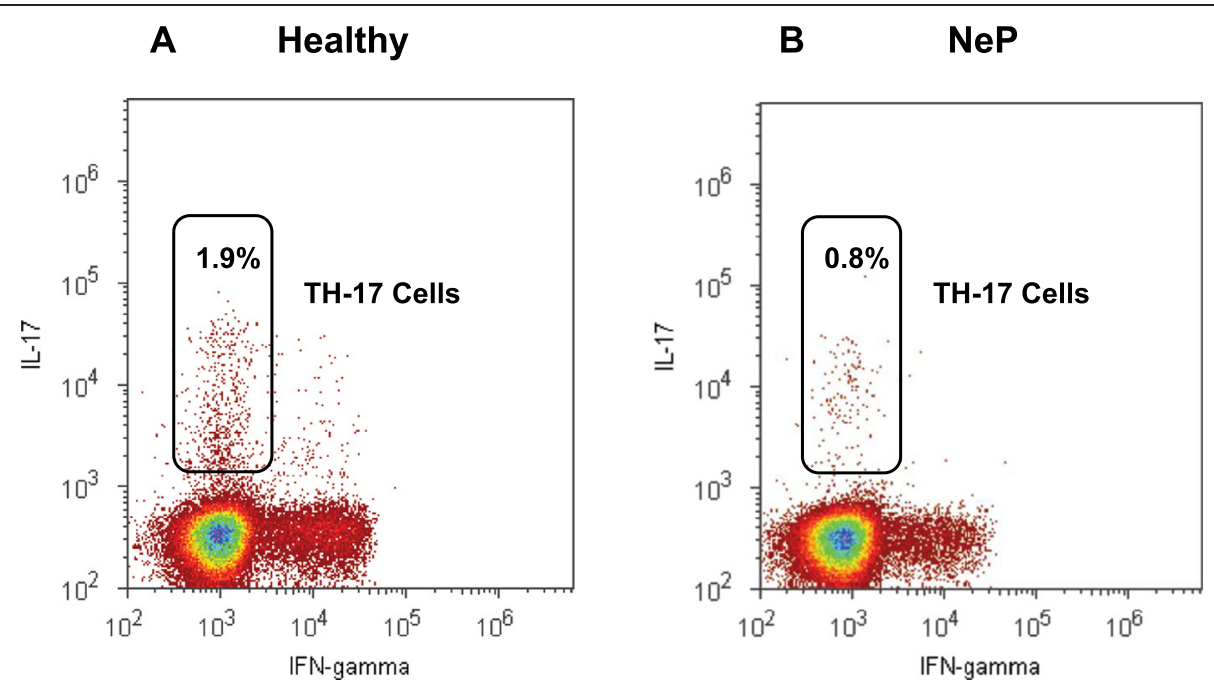

Figure 1 Gating strategy for the detection of pro-inflammatory TH17 cells. PBMCs were stained with Brilliant Violet (BV421)-labeled anti-human IL-17 antibody. Lymphocyte population was gated from PBMCs according to forward scatter (FSC) characteristics and side scatter (SSC) characteristics (see Figure 2) and then separated in $\mathrm{LL}-17^{+} \mathrm{TH} 17$ cells. Representative results of a healthy control with a higher number of TH17 cells (A) and a patient suffering from neuropathic pain with less TH17 cells (B) are shown. TH, T helper cells; PBMC, Peripheral blood mononuclear cells; IL, Interleukin.

false discovery level of $\mathrm{q}<0.05$, and $P$ values were adjusted accordingly following the Benjamini-Hochberg algorithm. $P<0.05$ were considered to be statistically significant. Results are expressed as mean \pm standard deviation (SD) in the text.

\section{Results}

\section{Subjects}

Within two years of recruitment, 26 patients fulfilling the inclusion criteria and 26 healthy controls were enrolled. The characteristics of the participating patients and pain syndromes are given in Table 2.

\section{Granulocytes and lymphocytes were only slightly changed in neuropathic pain}

Venous blood was drawn between 9:00 and 9:30 am into vacutainers containing Ethylenediaminetetraacetic acid (EDTA) for routine laboratory studies. Upon analyzing the number of neutrophil granulocytes, representing an essential part of the innate immune system, as well as lymphocytes, we found only slight alterations in patients with neuropathic pain (neutrophils: $55.4 \pm 9.1 \%$ in controls versus $58.6 \pm 9.3 \%$ in neuropathic pain, $P=0.268$; lymphocytes: $33.8 \pm 8.1 \%$ in controls versus $29.2 \pm 8.2 \%$ in neuropathic pain, $P=0.069$, Table 3 ).

\section{Cytokine measurement did not reveal relevant results}

The specific functions of T-cell subsets are based on their respective cytokine release. TH1 cells produce predominantly pro-inflammatory cytokines such as IFN- $\gamma$ and TNF- $\alpha$, which support cellular immunity, whereas TH2 cells release anti-inflammatory cytokines, including IL-4 and IL-10, which mediate humoral immunity. TH17 cells particularly produce the potent pro-inflammatory cytokine IL-17, which is involved in many inflammatory conditions. IL-23 is a key cytokine in the control of inflammation in peripheral tissues, which stimulates naïve CD4 T-cells to differentiate into TH17 cells, in conjunction with IL- 6 and TGF- $\beta$. Tregs have an antiinflammatory role by releasing anti-inflammatory cytokines like IL-10 and TGF- $\beta$. However, it seems likely, that TH1 and TH17 cytokines are central to increased pain sensitivity, whereas $\mathrm{TH} 2$ and Treg derived cytokines may be protective.

Therefore, we analyzed blood levels of pain-associated and T-cell-related cytokines using human cytokine multiplex immunoassay (MIP- $1 \alpha$, TNF- $\alpha$, IFN- $\gamma$, IL-4, IL-6, IL-10, IL-17, and IL-23). Serum levels of IL-4, TNF- $\alpha$ and IFN- $\gamma$ were neither detectable in the peripheral blood of patients nor in healthy controls. No differences between patients and healthy controls were found analyzing IL-6, IL-10 and IL-17. In accordance with numerous described studies, serum levels of pro-inflammatory cytokines MIP$1 \alpha$ and IL-23 were significantly higher in the peripheral blood of patients suffering from neuropathic pain. However, it has to be noted that IL-23 was the only cytokine with values above the so-called LLOQ, the lowest concentration that can be reliably detected (see Methods section). (IL-6: $1.2 \pm 0.8 \mathrm{pg} / \mathrm{ml}$ in controls versus $2.5 \pm 2.4 \mathrm{pg} / \mathrm{ml}$ in neuropathic pain, $P=0.064$; IL-10: $3.56 \pm 2.45 \mathrm{pg} / \mathrm{ml}$ in controls versus $3.69 \pm 2.40 \mathrm{pg} / \mathrm{ml}$ in neuropathic pain, $P=0.84 ;$ IL-17: $3.53 \pm 2.11 \mathrm{pg} / \mathrm{ml}$ in controls versus $4.29 \pm 2.02 \mathrm{pg} / \mathrm{ml}$ in neuropathic pain, $P=0.23$; MIP- $1 \alpha$ : $17.2 \pm 11.2 \mathrm{pg} / \mathrm{ml}$ in controls versus $28.4 \pm 16.4 \mathrm{pg} / \mathrm{ml}$ in 


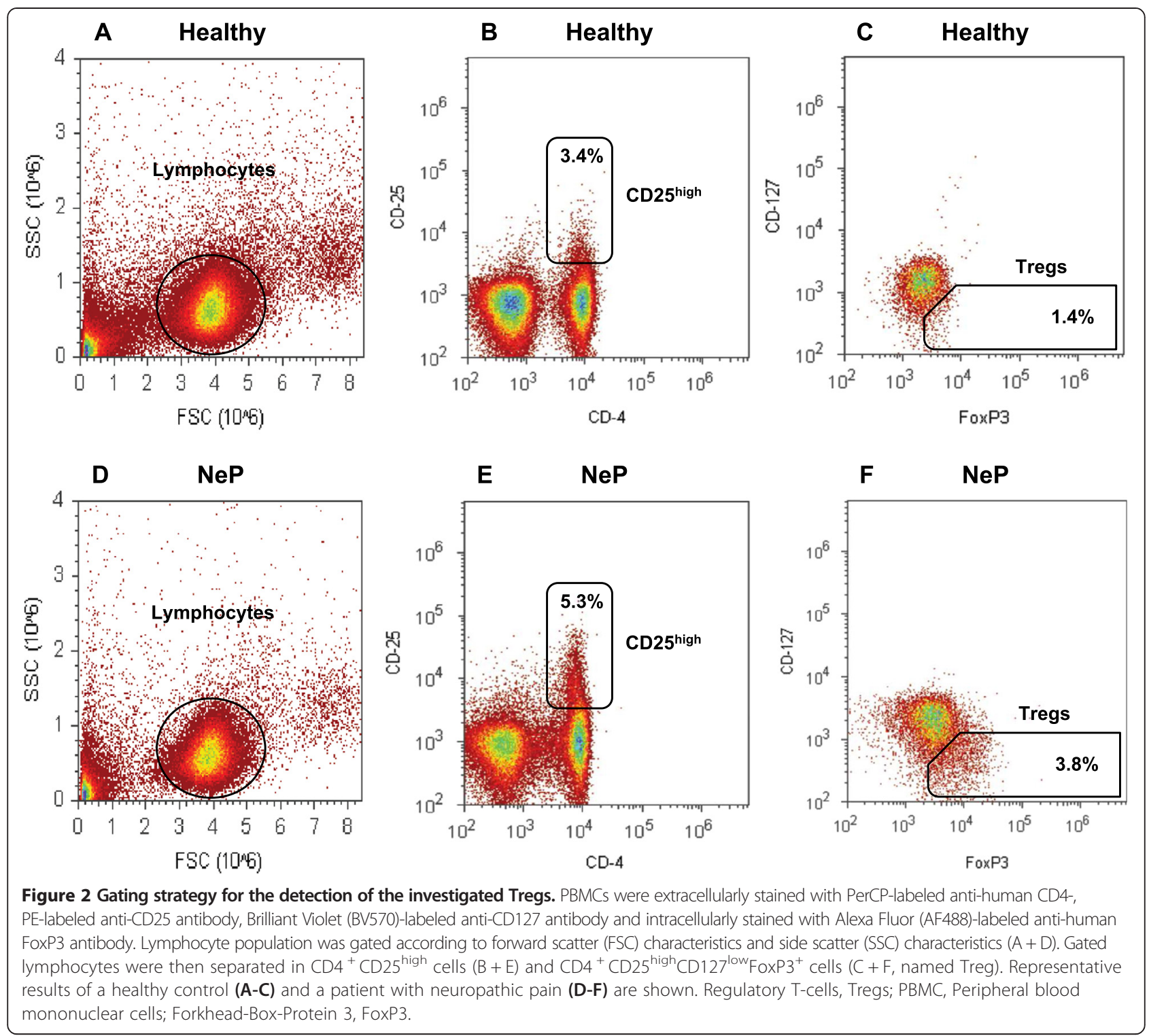

Table 1 RT-PCR assay characteristics and primer sequences

\begin{tabular}{ll}
\hline Gene & Primer sequence \\
\hline SDHA & Roche RealTime Ready Single Assay ID 102136 \\
TBP & Roche RealTime Ready Single Assay ID 101145 \\
FoxP3 & Roche RealTime Ready Single Assay ID 113503 \\
TGF- $\beta$ & for 5'ACTACTACGCCAAGGAGGTCAC 3' \\
& rev 5'TGCTTGAACTTGCATAGATTTCG 3', UPL probe \#31 \\
RORYT & for 5'CAGCGCTCCAACATCTTCT 3' \\
& rev 5'CCACATCTCCCACATGGAC 3', UPL probe \#69
\end{tabular}

Table 2 Patient characteristics

\begin{tabular}{llllll}
\hline Item & Healthy & NeP (all) & PNeP & PHN & OFP \\
\hline Numbers (n) & 26 & 26 & 13 & 7 & 6 \\
Age & $39 \pm 11$ & $56 \pm 14^{*}$ & $49 \pm 14^{*}$ & $71 \pm 6^{*}$ & $53 \pm 10^{*}$ \\
Female & $52 \%$ & $73 \%$ & $69 \%$ & $71 \%$ & $83 \%$ \\
BMI & $23.4 \pm 2.8$ & $24.0 \pm 3.4$ & $25.1 \pm 3.2$ & $22.4 \pm 3.4$ & $23.6 \pm 3.8$ \\
NRS (rest) & $0.0 \pm 0.0$ & $4.5 \pm 2.3^{*}$ & $4.3 \pm 2.5^{*}$ & $3.7 \pm 1.6^{*}$ & $5.6 \pm 2.1^{*}$ \\
NRS (motion) & $0.0 \pm 0.0$ & $6.2 \pm 2.7^{*}$ & $6.0 \pm 2.3^{*}$ & $4.7 \pm 3.9^{*}$ & $6.6 \pm 3.3^{*}$ \\
KAB & $1.9 \pm 0.7$ & $3.2 \pm 0.8^{*}$ & $3.3 \pm 0.7^{*}$ & $2.9 \pm 1.1^{*}$ & $3.1 \pm 0.8^{*}$ \\
\hline
\end{tabular}

$\mathrm{NeP}$ (all): all patients suffering from neuropathic pain; PNeP: Peripheral neuropathic pain (symmetrical polyneuropathy/peripheral mononeuropathy); PHN: postherpetic neuralgia; OFP: orofacial pain; NRS (rest/motion): Numeric rating scale (0 to 10) of pain, 0 : 'no pain', 10: 'worst pain imaginable'; KAB: Questionnaire for self-perceived stress ranging from one (no stress) to six (maximum stress). Results are expressed as mean \pm standard deviation (SD), ${ }^{*} P<0.05$ versus healthy controls. 
Table 3 Differential blood count, flow cytometric and RT-PCR results of patient subgroups

\begin{tabular}{|c|c|c|c|c|c|}
\hline Item & Healthy & $\mathrm{NeP}$ (all) & $\mathrm{PNeP}$ & PHN & OFP \\
\hline Neutrophils (\%) & $55.4 \pm 9.1$ & $58.6 \pm 9.3$ & $57.5 \pm 10.1$ & $57.3 \pm 9.0$ & $62.3 \pm 8.5$ \\
\hline Lymphocytes (\%) & $33.8 \pm 8.1$ & $29.2 \pm 8.2$ & $30.5 \pm 7.9$ & $28.3 \pm 9.6$ & $27.2 \pm 8.1$ \\
\hline $\mathrm{CD}^{+}$Counts $\times 1000$ & $28.7 \pm 7.3$ & $33.5 \pm 15.4$ & $36.8 \pm 18.8$ & $30.3 \pm 15.3$ & $30.8 \pm 2.9$ \\
\hline $\mathrm{CD}^{+}(\%)$ & $43.4 \pm 9.9$ & $47.5 \pm 11.9$ & $49.9 \pm 12.6$ & $44.3 \pm 13.8$ & $46.6 \pm 8.0$ \\
\hline TH1 (\%) & $9.7 \pm 4.7$ & $9.6 \pm 4.1$ & $10.9 \pm 4.7$ & $7.2 \pm 3.1$ & $9.3 \pm 2.7$ \\
\hline TH2 (\%) & $1.3 \pm 1.2$ & $1.5 \pm 0.8$ & $1.7 \pm 0.7$ & $0.9 \pm 0.5$ & $2.0 \pm 0.9$ \\
\hline TH17 (\%) & $1.3 \pm 1.0$ & $0.7 \pm 0.4^{*}$ & $0.9 \pm 0.4$ & $0.4 \pm 0.1^{*}$ & $0.8 \pm 0.5$ \\
\hline RORYT & $2.7 \pm 1.4$ & $1.9 \pm 1.0$ & $1.8 \pm 0.6^{*}$ & $1.9 \pm 1.2^{*}$ & $2.2 \pm 1.5$ \\
\hline $\mathrm{CD} 4^{+} \mathrm{CD} 25^{\text {high }}(\%)$ & $3.7 \pm 0.7$ & $5.4 \pm 1.5^{*}$ & $5.1 \pm 1.8^{*}$ & $5.0 \pm 0.7^{*}$ & $6.4 \pm 0.9^{*}$ \\
\hline Tregs (\%) & $2.0 \pm 1.0$ & $3.9 \pm 1.3^{*}$ & $3.5 \pm 1.1^{*}$ & $4.0 \pm 1.9^{*}$ & $4.4 \pm 0.9^{*}$ \\
\hline FoxP3 & $0.6 \pm 0.2$ & $1.2 \pm 0.8^{*}$ & $1.0 \pm 0.7^{*}$ & $1.3 \pm 0.3^{*}$ & $1.4 \pm 1.3^{*}$ \\
\hline
\end{tabular}

$\mathrm{NeP}$ (all): all patients suffering from neuropathic pain; PNeP: Peripheral neuropathic pain (symmetrical polyneuropathy/peripheral mononeuropathy);

PHN: postherpetic neuralgia; OFP: orofacial pain; Results are expressed as mean \pm standard deviation (SD), ${ }^{*} P<0.05$ versus healthy controls.

neuropathic pain, $P=0.022$; IL-23: $0.9 \pm 0.3 \mathrm{pg} / \mathrm{ml}$ in controls versus $1.2 \pm 0.4 \mathrm{pg} / \mathrm{ml}$ in neuropathic pain, $P=0.022$ ).

TH17 frequency was distinctly decreased in neuropathic pain Although many studies have analyzed the role of TH17 cells in human autoimmune diseases, there are very limited data on the role of TH17 cells in patients with neuropathic pain. TH17 cells act as an important pro-inflammatory component and have been shown to promote inflammation in a number of diseases. The proportion of TH17 cells is expressed as percentage of all T-cells. As shown in Figure 3, the frequency of TH17 cells was evidently decreased in the peripheral blood of patients suffering from neuropathic pain. Affirming these results, the relative mRNA expression of the TH17 cell-specific transcription factor ROR $\gamma \mathrm{T}$ was reduced, but did not reach significance (TH17 cells: $1.3 \pm 1.0 \%$ in controls versus $0.7 \pm 0.4 \%$ in neuropathic pain, $P=0.046$; relative ROR $\gamma \mathrm{T}$ mRNA expression: $2.7 \pm 1.4$ in controls versus $1.9 \pm 1.0$ in neuropathic pain, $P=0.064$; Figure 3 ).

Treg frequency was distinctly increased in neuropathic pain Human regulatory $\mathrm{T}$-cells play a vital role in controlling the adaptive immune response and in maintaining self-

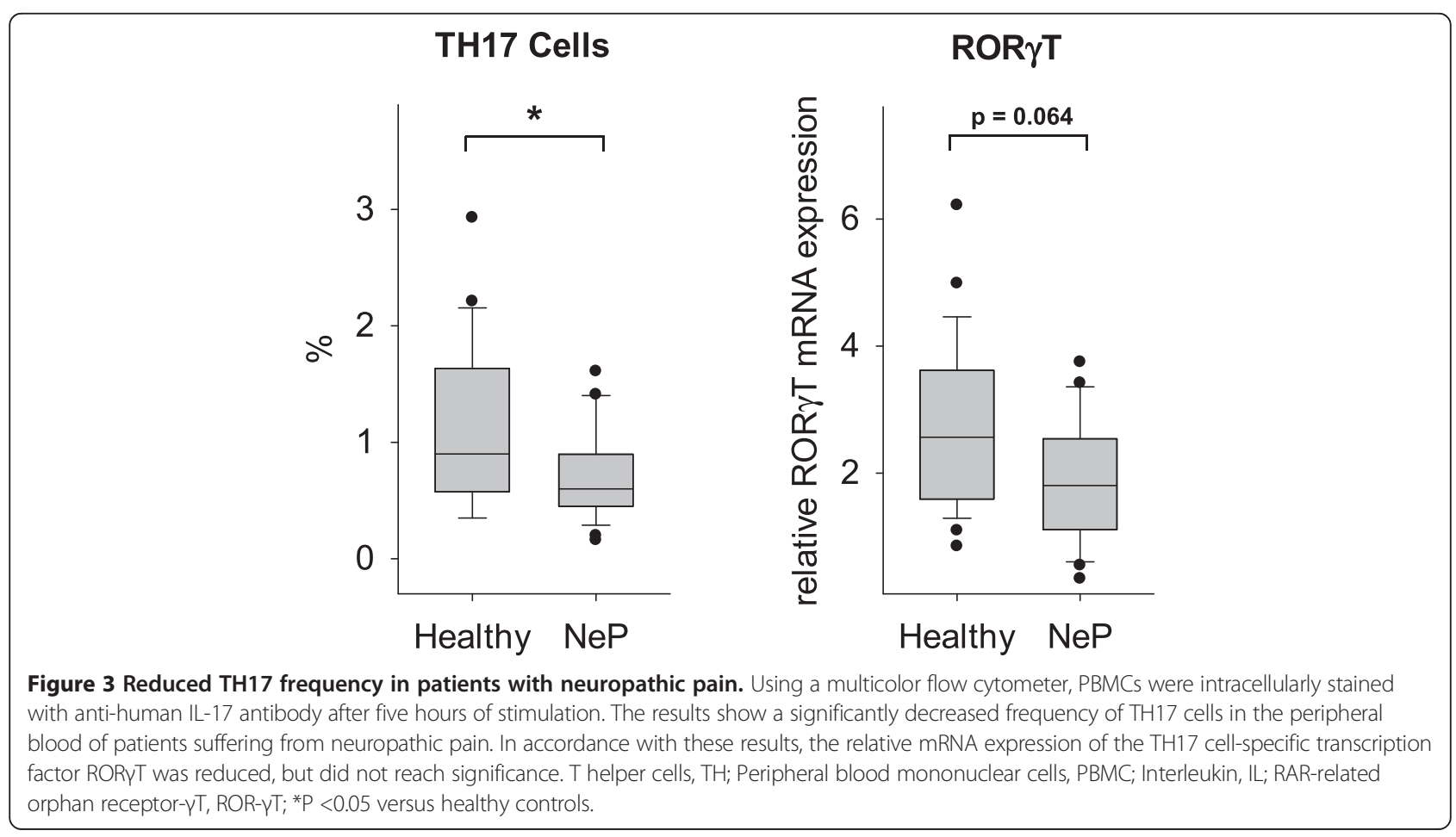


tolerance. Tregs have been shown to prevent autoimmune diseases and to limit chronic inflammatory and nervous system disturbances. On the other hand, the strong Treginduced immune suppression also impairs beneficial responses such as anti-tumor immunity $[27,28]$. However, there were limited data analyzing the functional role of Tregs in neuropathic pain, which therefore remained to be investigated. We analyzed the number of Tregs by flow cytometry, using two staining procedures; classic extracellular staining with $\mathrm{CD} 4^{+} \mathrm{CD} 25^{\text {high }}$ and the more specific intra- and extracellular staining procedure with $\mathrm{CD} 4{ }^{+} \mathrm{CD} 25^{\text {high }} \mathrm{CD} 127^{\text {low }} \mathrm{FoxP} 3^{+}$. We defined $\mathrm{CD} 44^{+} \mathrm{CD} 25^{\text {high }} \mathrm{CD} 127^{\text {low }}{ }^{\text {FoxP3 }}{ }^{+}$T-cells as Tregs. The prevalence of Tregs was expressed as a ratio of $\mathrm{CD} 4{ }^{+} \mathrm{CD} 25^{\text {high }} \mathrm{CD} 127^{\text {low }}$ FoxP3 ${ }^{+} \mathrm{T}$ cells as a percentage of $\mathrm{CD}^{+}{ }^{+}$T-cells. Figure 3 shows a significantly increased frequency of Tregs in patients with neuropathic pain as compared to controls. To confirm the quantitative observations of the Treg frequency we also determined the relative mRNA expression of the specific transcription factor FoxP3 and TGF- $\beta$ by quantitative real-time PCR (qPCR). As shown on Figure 4, significantly increased mRNA levels of FoxP3 and TGF- $\beta$ were observed in patients with neuropathic pain. These results were consistent with the flow cytometric analyses (Tregs: $2.0 \pm 1.0 \%$ in controls versus $3.9 \pm 1.3 \%$ in neuropathic pain, $P=0.007$; relative FoxP3 mRNA expression: $0.6 \pm 0.2$ in controls versus $1.2 \pm 0.8$ in neuropathic pain, $P=0.028$; relative TGF- $\beta$ mRNA expression: $0.15 \pm 0.06$ in controls versus $0.25 \pm 0.15$ in neuropathic pain, $P=0.009$; Figure 4 ).

\section{TH1/TH2 balance was only slightly altered in neuropathic pain}

In previous investigations, the ratio of $\mathrm{TH} 1$ and $\mathrm{TH} 2$ cells was used to characterize immune responses in different diseases. In the present study, a trend towards a decreased TH1/TH2 ratio was observed, which, however, did not reach significance (TH1/TH2: $16.1 \pm 17.4$ in controls versus $10.1 \pm 10.0$ in neuropathic pain, $P=0.56$; Figure 5 ).

\section{TH17/Treg balance was markedly disrupted in neuropathic pain}

TH17 cells play an important pro-inflammatory role whereas Tregs are strong immune suppressors. Therefore, the balance between TH17 cells and Tregs, along with $\mathrm{TH} 1 / \mathrm{TH} 2$ balance, is an important factor in analyzing the immune response. Our results regarding $\mathrm{T}$-cell subsets alone demonstrated markedly reduced pro-inflammatory TH17 cells with simultaneous elevated anti-inflammatory Tregs. Conclusively, as shown in Figure 5, the TH17/Treg ratio was significantly lower in the peripheral blood of patients compared to healthy controls. These results indicate a clear anti-inflammatory T-cell shift in neuropathic pain (TH17/Treg: $0.9 \pm 1.1$ in controls versus $0.2 \pm 0.1$ in neuropathic pain, $P<0.007$; Figure 5).

\section{Discussion}

Neuropathic pain is a severe and frequent condition which affects up to $18 \%$ of the population [29]. The pathophysiological mechanisms leading to chronification of neuropathic pain are a major focus of interest, but are 


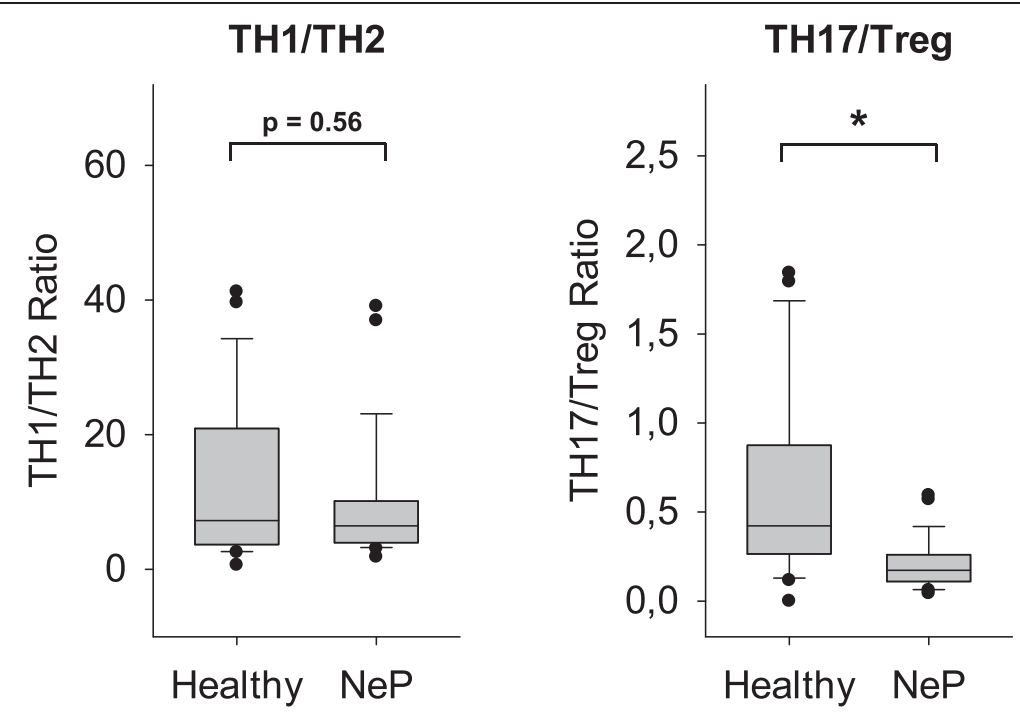

Figure 5 Altered $\mathrm{TH} 1 / \mathrm{TH} 2$ and $\mathrm{TH} 17 / \mathrm{Treg}$ balances in patients with neuropathic pain. While the $\mathrm{TH} 1 / \mathrm{TH} 2$ ratio was only slightly decreased, the $\mathrm{TH} 17 /$ Treg balance was significantly enhanced in patients as compared to healthy controls. These results indicate a clear anti-inflammatory T-cell shift in neuropathic pain. T helper cell, TH; Regulatory T-cell, Treg; ${ }^{*} \mathrm{P}<0.05$ versus healthy controls.

not yet completely elucidated. Recent data indicate a critical involvement of the innate and adaptive immune system in the pathophysiology of chronification. Several types of immune cells have been implicated in the pathogenesis of neuropathic pain [3]. The innate immune system has been shown to be important during the early stages of acute pain, represented particularly by neutrophils [13]. Regarding chronification, T-lymphocytes, as key players of the adaptive immune system, seem to be of major importance [30,31]. Traditionally, it has been suggested that neuropathic pain is associated with a pro-inflammatory immune response. Therefore, mainly anti-inflammatory treatments targeting cytokines and immune cells have been evaluated in several animal models of neuropathic pain [3]. In a recent study, neuropathic pain induced by experimental autoimmune neuritis was successfully attenuated by expanding Tregs [17]. In humans, the first Treg-expanding tests were stopped because of life-threatening side effects [19]. Nevertheless, the modulation of T-cells is still the focus of intense research [32].

In the present study, we analyzed the pain-related cytokines MIP- $1 \alpha$, TNF- $\alpha$, IFN- $\gamma$, IL- 4 , IL-6, IL-10, IL-17 and IL-23 in the peripheral blood of 26 patients with neuropathic pain and compared the results with those of 26 healthy controls. We found that the serum levels of IL-4, TNF- $\alpha$ and IFN- $\gamma$ were below the detectable limit and no differences were found regarding IL-6, IL-10 and IL-17. Only the pro-inflammatory cytokines MIP- $1 \alpha$ and IL-23 were significantly higher in neuropathic pain. It has to be noted though, that except for IL-23, values of all cytokines measured were below the lowest concentration of an analyte in a sample that can be reliably detected. This shortcoming of serum cytokine measurements in pain syndromes has also been described by other authors [33]. Taken together, our results indicated that serum cytokine levels alone are not sufficient to monitor the adaptive immune response in neuropathic pain and led us to analyze the cellular compartment.

By routine laboratory studies regarding the differential leucocyte count, we found only an unchanged number of neutrophil granulocytes and a slightly reduced number of lymphocytes in the peripheral blood of patients with neuropathic pain. Using multicolor flow cytometry, we subsequently quantified the numbers of $\mathrm{TH} 1, \mathrm{TH} 2$, TH17 and Treg cells in the peripheral blood of our patients. Contrary to our initial assumption, we found clear indications for an anti-inflammatory T-cell shift: Pro-inflammatory TH17 cells were significantly decreased, whereas anti-inflammatory Tregs were significantly increased. Consequently, the corresponding TH17/Treg ratio was distinctly shifted towards an anti-inflammatory immune response. To confirm the quantitative observations of the TH17 and Treg frequency, we also determined the relative mRNA expression of the TH17 cell-specific transcription factor ROR $\gamma \mathrm{T}$, as well as TGF- $\beta$ and the Treg-specific transcription factor FoxP3 by quantitative real-time PCR. A diminished ROR $\mathrm{T}$ T mRNA expression was in line with the reduced number of TH17 cells, while a notably elevated FoxP3 and TGF- $\beta$ mRNA expression confirmed the increased Treg frequency. Regarding patient subgroups, we found no differences in respect of the anti-inflammatory T-cell shift and mRNA expressions between patients suffering from orofacial pain, postherpetic 
neuralgia and other types of peripheral neuropathies (Table 3). The question arises whether the observed changes are of clinical relevance, particularly in view of the overall low portion of the specific T-cell subsets. Furthermore, it would be interesting and relevant to investigate whether the immune changes can also be found in the affected tissue.

Our results are, at first glance, unexpected since the majority of previously published data describes the association between pain and 'immune activation' based on investigations of TH1 and TH2 cells, as well as cytokines. This previous TH1/TH2 paradigm has, however, been revised and updated with the discovery of TH17 cells and the more specific detection of Tregs. Our findings, together with recently published data regarding various T-cell subsets, point to a strong association between pain and 'immune suppression'. Interestingly, the T-cell response in the present study is comparable with our recent findings in patients with CLBP, who also presented with high pain and stress levels, but had no signs of neuropathic pain [20]. There is a general consensus that neuropathic and nociceptive pain are distinct entities, although basic research clearly reveals a huge overlap of underlying pathophysiological mechanisms, including neurotransmitters and cytokines [2]. Our results show for the first time that in both neuropathic and nociceptive pain the adaptive immune system is altered in the same anti-inflammatory way. The context of chronic stress and immune suppression has been described for many years, although not extensively with regard to TH17 cells and Tregs [34]. An anti-inflammatory T-cell shift has been found in patients with chronic mild depression or chronic fatigue syndrome [35-37], and both disorders are frequently associated with all types of chronic pain.

Concerning the cellular mechanisms, T-cell differentiation mainly depends on the cytokine milieu of the microenvironment, but other pathways have also been shown to be involved. For example, the hypothalamicpituitary-adrenal axis mediates immune regulation through binding of stress hormones like adrenocorticotropic hormone or cortisol to their cognate receptors at the surface of T-cells. Furthermore, the sympathetic nervous system is known to induce immune dysregulation via adrenaline and noradrenaline [38]. These processes in turn play an important role in negative emotional states, such as stress and depression. Our patients with neuropathic pain also suffered from stress and psychological burden as revealed by the enhanced KAB values. We therefore hypothesize that the altered immune responses in both of our studies might reflect a particular chronic pain-related stress reaction

\section{Conclusions}

In summary, we found a TH17/Treg imbalance with increased anti-inflammatory Tregs and decreased pro- inflammatory TH17 cells in patients with neuropathic pain. These results are quite similar to our previous findings in patients with nociceptive CLBP who did not show any signs of neuropathy, but similar pain and stress levels. Therefore, it remains to be clarified in future studies whether the immune changes represent an underlying pathophysiological mechanism or an epiphenomenon induced by ongoing pain and stress.

\section{Additional files}

Additional file 1: Figure S1. Exemplary density plots of 10 healthy controls and 10 patients with neuropathic pain showing pro-inflammatory TH17 cells.

Additional file 2: Figure S2. Exemplary density plots of 10 healthy controls and 10 patients with neuropathic pain showing anti-inflammatory Tregs.

\section{Abbreviations}

CLBP: Chronic low back pain; FACS: Fluorescent-activated cell sorting; RTPCR: Quantitative real-time PCR (qPCR); TH: T-helper cell; Treg: Regulatory Tcell; FoxP3: Forkhead-Box-Protein P3; TGF- $\beta$ : Transforming growth factor- $\beta$; RORYT: RAR-related orphan receptoryT; MIP1-a: Macrophage inflammatory protein 1-a; TNF-a: Tumor necrosis factor-a; IFN: Interferon; IL: Interleukin; KAB: German version of the Questionnaire for Actual Demands

"Kurzfragebogen zur aktuellen Beanspruchung".

\section{Competing interests}

The authors declare that they have no competing interests.

\section{Authors' contributions}

All authors read and approved the final manuscript. BL performed all experiments and wrote the manuscript. BL and BRA recruited the patients and prepared the blood samples. BL, BRA, JH, SK and SCA analyzed the data. $\mathrm{BL}, \mathrm{BRA}$ and SCA designed the experiments.

\section{Acknowledgements}

The authors are grateful to G Groeger and J Rink for excellent technical assistance.

This work was supported by grants from the Hella-Langer-Stiftung, Germany.

Received: 22 August 2014 Accepted: 18 December 2014

Published online: 21 January 2015

\section{References}

1. Treede RD, Jensen TS, Campbell JN, Cruccu G, Dostrovsky JO, Griffin JW, et al. Neuropathic pain: redefinition and a grading system for clinical and research purposes. Neurology. 2008;70(18):1630-5.

2. Cohen SP, Mao J. Neuropathic pain: mechanisms and their clinical implications. BMJ. 2014;348:f7656.

3. Austin PJ, Moalem-Taylor G. The neuro-immune balance in neuropathic pain: involvement of inflammatory immune cells, immune-like glial cells and cytokines. J Neuroimmunol. 2010;229(1-2):26-50

4. Tian L, Ma L, Kaarela T, Li Z. Neuroimmune crosstalk in the central nervous system and its significance for neurological diseases. J Neuroinflammation. 2012;9:155.

5. Iwatsuki K, Arai T, Ota H, Kato S, Natsume T, Kurimoto S, et al. Targeting anti-inflammatory treatment can ameliorate injury-induced neuropathic pain. PLoS One. 2013;8(2):e57721.

6. Guptarak J, Wanchoo S, Durham-Lee J, Wu Y, Zivadinovic D, Paulucci-Holthauzen A, et al. Inhibition of IL-6 signaling: a novel therapeutic approach to treating spinal cord injury pain. Pain. 2013;154(7):1115-28.

7. Kiguchi N, Maeda T, Kobayashi Y, Fukazawa Y, Kishioka S. Macrophage inflammatory protein-1alpha mediates the development of neuropathic pain following peripheral nerve injury through interleukin-1 beta up-regulation. Pain. 2010;149(2):305-15. 
8. Kim CF, Moalem-Taylor G. Interleukin-17 contributes to neuroinflammation and neuropathic pain following peripheral nerve injury in mice. J Pain. 2011;12(3):370-83.

9. Shi X, Chen Y, Nadeem L, Xu G. Beneficial effect of TNF-alpha inhibition on diabetic peripheral neuropathy. J Neuroinflammation. 2013;10:69.

10. Cunha FQ, Poole S, Lorenzetti BB, Veiga FH, Ferreira SH. Cytokine-mediated inflammatory hyperalgesia limited by interleukin-4. Br J Pharmacol. 1999;126 (1):45-50.

11. Plunkett JA, Yu CG, Easton JM, Bethea JR, Yezierski RP. Effects of interleukin-10 (IL-10) on pain behavior and gene expression following excitotoxic spinal cord injury in the rat. Exp Neurol. 2001;168(1):144-54.

12. Echeverry S, Shi XQ, Haw A, Liu H, Zhang ZW, Zhang J. Transforming growth factor-beta1 impairs neuropathic pain through pleiotropic effects. Mol Pain. 2009;5:16.

13. Perkins NM, Tracey DJ. Hyperalgesia due to nerve injury: role of neutrophils. Neuroscience. 2000;101(3):745-57.

14. Liu HH, Xia XY, Wu YM, Pan $\sqcup$, Jin BF, Shang XJ, et al. Detection of peripheral blood Th1/Th2 cell ratio in patients with chronic abacterial prostatitis/chronic pelvic pain syndrome. Zhonghua Nan Ke Xue. 2006;12(4):330-2. 336

15. Schinkel C, Gaertner A, Zaspel J, Zedler S, Faist E, Schuermann M. Inflammatory mediators are altered in the acute phase of posttraumatic complex regional pain syndrome. Clin J Pain. 2006;22(3):235-9.

16. Kleinschnitz C, Hofstetter HH, Meuth SG, Braeuninger S, Sommer C, Stoll G. T cell infiltration after chronic constriction injury of mouse sciatic nerve is associated with interleukin-17 expression. Exp Neurol. 2006;200(2):480-5.

17. Austin PJ, Kim CF, Perera CJ, Moalem-Taylor G. Regulatory T cells attenuate neuropathic pain following peripheral nerve injury and experimental autoimmune neuritis. Pain. 2012;153(9):1916-31.

18. Xing Q, Hu D, Shi F, Chen F. Role of regulatory T cells in patients with acute herpes zoster and relationship to postherpetic neuralgia. Arch Dermatol Res. 2013;305(8):715-22.

19. Suntharalingam G, Perry MR, Ward S, Brett SJ, Castello-Cortes A, Brunner $M D$, et al. Cytokine storm in a phase 1 trial of the anti-CD28 monoclonal antibody TGN1412. N Engl J Med. 2006;355(10):1018-28.

20. Luchting B, Rachinger-Adam B, Zeitler J, Egenberger L, Mohnle P, Kreth S, et al. Disrupted TH17/Treg balance in patients with chronic low back pain. PLoS One. 2014;9(8):e104883.

21. Freynhagen R, Baron R, Gockel U. Tolle TR. painDETECT: a new screening questionnaire to identify neuropathic components in patients with back pain. Curr Med Res Opin. 2006;22(10):1911-20

22. Maier C, Baron R, Tolle TR, Binder A, Birbaumer N, Birklein F, et al. Quantitative sensory testing in the German Research Network on Neuropathic Pain (DFNS): somatosensory abnormalities in 1236 patients with different neuropathic pain syndromes. Pain. 2010;150(3):439-50

23. Gauter-Fleckenstein B, Kaviani R, Weiss C, Burges A, Korell M, Anthuber C, et al. Perioperative patient management. Evaluation of subjective stress and demands of patients undergoing elective gynaecological surgery. Anaesthesist. 2007:56(6):562-70

24. Chowdhury F, Williams A, Johnson P. Validation and comparison of two multiplex technologies, Luminex and Mesoscale Discovery, for human cytokine profiling. J Immunol Methods. 2009;340(1):55-64

25. Venet F, Malcus C, Ferry T, Poitevin F, Monneret G. Percentage of regulatory T cells CD4 + CD25 + CD127- in HIV-infected patients is not reduced after cryopreservation. J Immunol Methods. 2010;357(1-2):55-8.

26. Ledderose $\mathrm{C}$, Heyn J, Limbeck E, Kreth S. Selection of reliable reference genes for quantitative real-time PCR in human T cells and neutrophils. BMC Res Notes. 2011:4:427.

27. Whiteside TL. Disarming suppressor cells to improve immunotherapy. Cancer Immunol Immunother. 2012;61(2):283-8.

28. Tao H, Mimura Y, Aoe K, Kobayashi S, Yamamoto H, Matsuda E, et al. Prognostic potential of FOXP3 expression in non-small cell lung cancer cells combined with tumor-infiltrating regulatory T cells. Lung Cancer. 2012;75(1):95-101.

29. Toth C, Lander J, Wiebe S. The prevalence and impact of chronic pain with neuropathic pain symptoms in the general population. Pain Med 2009;10(5):918-29.

30. Hu P, Bembrick AL, Keay KA, McLachlan EM. Immune cell involvement in dorsal root ganglia and spinal cord after chronic constriction or transection of the rat sciatic nerve. Brain Behav Immun. 2007;21(5):599-616.

31. Costigan M, Moss A, Latremoliere A, Johnston C, Verma-Gandhu M, Herbert TA, et al. T-cell infiltration and signaling in the adult dorsal spinal cord is a major contributor to neuropathic pain-like hypersensitivity. J Neurosci. 2009;29(46):14415-22.

32. Sakaguchi S, Miyara M, Costantino CM, Hafler DA. FOXP3+ regulatory T cells in the human immune system. Nat Rev Immunol. 2010;10(7):490-500.

33. Lenz M, Uceyler N, Frettloh J, Hoffken O, Krumova EK, Lissek S, et al. Local cytokine changes in complex regional pain syndrome type I (CRPS I) resolve after 6 months. Pain. 2013;154(10):2142-9.

34. Segerstrom SC, Miller GE. Psychological stress and the human immune system: a meta-analytic study of 30 years of inquiry. Psychol Bull. 2004;130(4):601-30.

35. Torres-Harding S, Sorenson M, Jason LA, Maher K, Fletcher MA. Evidence for T-helper 2 shift and association with illness parameters in chronic fatigue syndrome (CFS). Bull IACFS ME. 2008;16(3):19-33.

36. Raison $\mathrm{CL}$, Miller AH. Is depression an inflammatory disorder? Curr Psychiatry Rep. 2011;13(6):467-75.

37. Hong M, Zheng J, Ding ZY, Chen JH, Yu L, Niu Y, et al. Imbalance between Th17 and Treg cells may play an important role in the development of chronic unpredictable mild stress-induced depression in mice. Neuroimmunomodulation. 2013;20(1):39-50

38. Glaser R, Kiecolt-Glaser JK. Stress-induced immune dysfunction: implications for health. Nat Rev Immunol. 2005:5(3):243-51.

\section{Submit your next manuscript to BioMed Central and take full advantage of:}

- Convenient online submission

- Thorough peer review

- No space constraints or color figure charges

- Immediate publication on acceptance

- Inclusion in PubMed, CAS, Scopus and Google Scholar

- Research which is freely available for redistribution

Submit your manuscript at www.biomedcentral.com/submit 